\title{
A Novel Multiobjective Framework for Cell Switch-Off in Dense Cellular Networks
}

\author{
David González $\mathrm{G}^{1}$, Halim Yanikomeroglu ${ }^{2}$, Mario Garcia-Lozano ${ }^{1}$ and Silvia Ruiz Boqué ${ }^{1}$ \\ ${ }^{1}$ Department of Signal Theory and Communications \\ Universitat Politècnica de Catalunya, Barcelona, Spain. \\ ${ }^{2}$ Department of Systems and Computer Engineering \\ Carleton University, Ottawa, Canada. \\ Emails: david.gonzalez.g@ieee.org, halim@sce.carleton.ca, \{mariogarcia, silvia\}@tsc.upc.edu
}

\begin{abstract}
The green communications paradigm has been receiving much attention in wireless networks in recent years. More specifically, in the context of cellular communications, the concept of Cell Switch Off (CSO) has been recognized as a promising approach to reduce the energy consumption. The need is expected to be pressing especially in the next decade with the increasing small cell deployment. However, the cell switch on/off decisions compounded by the resource allocation task in CSO constitute a highly challenging optimization problem due to the fact that this problem can be viewed as a generalized version of the resource allocation (scheduling) problem in the conventional cellular networks without CSO, which itself is already difficult. This paper introduces a novel framework to CSO based on multiobjective evolutionary optimization.

The main contribution of this paper is that the proposed multiobjective framework takes the traffic behaviour in both space and time (known by operators) into account in the optimal cell switch on/off decision making which is entangled with the corresponding resource allocation task. The exploitation of this statistical information is done in a number of ways, including through the introduction of a weighted network capacity metric. This indicator prioritizes cells which are expected to have traffic concentration resulting in on/off decisions that achieve substantial energy savings in scenarios where traffic is highly unbalanced, without compromising the QoS. The proposed framework distinguishes itself from the CSO papers in the literature in two ways: 1) The number of cell switch on/off transitions as well as handoffs are minimized. 2) The computationally-heavy part of the algorithm is executed offline, which makes the real-time implementation feasible.
\end{abstract}

\section{INTRODUCTION}

Cellular communications have grown exponentially worldwide. The rising cost of energy and the increased environmental awareness have created the urgent need for developing energy efficient green communications [1].

According to [2], mobile devices are becoming the preferred way to access the Internet, powered by the increasingly growing market of web-enabled mobile devices including smarter phones, tablets, and notebooks. The situation is far from helping to reduce the energy consumption in cellular networks as mobile operators are increasing the number of base station to cope with this explosive demand of data-based services and applications. Thus, academia and industry are investing big efforts in developing energy efficient networks.

Initial approaches were oriented towards minimizing the transmitted power over the air interface which in turn reduces the electromagnetic pollution and its potential risks on human health. Unfortunately, between $50 \%$ to $80 \%$ of the energy consumption in cellular networks takes place in the base stations [3]; this consumption is largely independent of traffic load. Therefore, the idea of switching off lightly loaded base stations has been considered recently as a promising alternative to reduce energy consumption in cellular networks. The framework is known as Cell Switch Off (CSO) and it is focused on determining the largest set of cells that can be switched off without compromising the Quality of Service (QoS) provided to users. This is the problem addressed in this paper. Fundamentally, the idea is to exploit temporal and spatial variations of traffic to turn off base stations in which a significant part of resources are idle. Recall that cellular networks are dimensioned to meet traffic demand in the busy hour, and hence, most of the resources are underutilized. Clearly, the dense cell deployment is the natural context to test the performance of CSO.

Given the relevance of the problem, the research on CSO schemes has been intensive in the last few years and numerous studies and proposals have been presented. Interesting theoretical analysis can be found in [4] and [5], where the authors showed the potential benefit of CSO in terms of energy saving and investigated the impact of other context variables such as site density and traffic load variability. However, the problem also has important practical aspects that must be considered including the impact of switch off/on transients (transitions), handover procedures, and implementation issues. Detailed discussions about the matter can be found in [6] and [7].

Inspired by this type of studies, several CSO schemes have been proposed. Most of them address the problem of selectively switching off base stations by means of heuristic algorithms. This schemes are preferred because CSO is a combinatorial NP-Complete problem, and hence, finding optimal solutions is not possible in polynomial time. Moreover, modeling intercell interference (ICI) accurately and the features found in realworld networks make the analysis even more complicated.

The paper is organized as follows: the next section presents a review of related literature and, in the light of the advantages and drawbacks of previous proposals, the novelty and contribution of this paper is indicated. The system model 
and multiobjective problem formulation are presented in Sections III and IV, respectively. The evaluation setting and the analysis of the results are shown in Section V. Finally, the paper is closed with the conclusions in Section VI.

\section{RELATED WORK AND NOVELTY}

Table I provides a comparison of several recent CSO proposals based on the following criteria:

- Architecture: Indicates whether the type of operation of each scheme is centralized, semi-distributed, or distributed.

- ICI: Indicates whether intercell interference is modeled realistically. This aspect is important because switching on/off cells modifies ICI levels significantly.

- Feasibility: Indicates whether implementation aspects and complexity issues are analyzed or taken into account. It includes the number of transitions/handovers, required intercell signalling and, real-time complexity.

- Coverage: Indicates whether each scheme considers coverage aspects such as the signal-to-interference-plus-noise ratio (SINR) or the receiver's sensitivity, i.e., minimum required power. Coverage analysis in CSO is crucial due to the fact that many coverage holes can appear as base stations are switched off.

As it can be seen, the majority of schemes require centralized operation. This is expected given the nature of CSO, i.e., a certain global knowledge is required in order to determine which base stations can be switched off. Although the centralized architectures may achieve a near-optimal performance, the feasibility is often a concern as the required amount of intercell signalling may be prohibitive. The semi-distributed and distributed schemes, on the other hand, are more feasible but, performance is typically much poorer than centralized approaches. In addition, convergence and stability are also common concerns. Note that ICI, the main capacity-limiting factor in cellular systems, is not modeled accurately in some proposals. Very often, full and constant ICI (worst case interference scenario) is assumed, which clearly hides the merit of those schemes and could lead to erroneous interpretations. Finally, coverage criteria are rarely taken into account. We believe that coverage criteria are important and must be included into the CSO picture. Although, coverage is considered initially during the planning stage of the network, switching off/on cells results in new cellular layouts, and hence, the initial coverage assessments are not longer valid. No mobile operator will be willing to modify their cellular layout without considering such a critical aspect. In the light of these observations, this paper presents a novel multiobjective framework that includes the strength of previous works but clearly differs from them in virtue of the following novelties:

1) The proposed scheme determines offline a set of different network configurations, each of them with different number of active cells, taking into account coverage criteria, average ICI levels, and spatial traffic distributions. Being the core processing executed offline, the approach is new in the sense that optimization is independent of
TABLE I

SUMMARY OF RELATED WORK.

\begin{tabular}{ccccc}
\hline Ref & Architecture & ICI & Feasibility & Coverage \\
\hline$[8]$ & Centralized & Partially & Partially & $\times$ \\
{$[9]$} & Centralized & $\checkmark$ & Partially & $\times$ \\
{$[10]$} & Centralized & $\checkmark$ & $\times$ & $\times$ \\
{$[11]$} & Cent/Dist & Partially & $\times$ & $\times$ \\
{$[12]$} & Centralized & $\checkmark$ & $\times$ & Partially \\
{$[13]$} & Semi-dist. & Partially & $\checkmark$ & $\times$ \\
{$[14]$} & Centralized & $\times$ & Partially & $\times$ \\
{$[15]$} & Centralized & Partially & Partially & $\times$ \\
{$[16]$} & Centralized & $\checkmark$ & Partially & $\times$ \\
{$[17]$} & Centralized & Partially & $\times$ & $\times$ \\
\hline
\end{tabular}

any particular realization of users, and so, results can be stored in look-up tables. Previous models operate based on every single (ephemeral) realization of users which requires a centralized and expensive processing.

2) The scheme allows a decoupled performance evaluation based on realistic system level simulations that can be flexibly set up according to operators' needs. Moreover, given that the results can be stored in databases, the scheme is suitable for a low real-time complexity implementation.

3) The set of traffic-profile-specific solutions are obtained by considering the tradeoff between aggregate network capacity and the number of switched off cells. These criteria are conflicting objectives, and hence, multiobjective optimization represents a convenient approach to investigate CSO through the Pareto dominance notion.

4) A novel metric called weighted network capacity is introduced in the context of CSO to estimate the fitness of each solution. This metric gives priority to cells in which the traffic is concentrated.

The proposed framework ${ }^{1}$ is explained in the next sections.

\section{System Model}

The system model is based on the downlink of an OFDMA cellular network. The system bandwidth is $B$ and the network is composed of $L$ cells. The coverage area is composed of $A$ small area elements (pixels). It is assumed that within each pixel, the average received power is constant, and hence, average SINR figures are also constant. The maximum transmit power per cell is $P_{\max }$.

Network geometry is given by the matrix $\mathbf{G} \in \mathbb{R}^{A \times L}$ containing the large scale statistics (path loss, antenna gain, and shadowing). The vectors $\mathbf{p}_{\mathrm{RS}}$ and $\mathbf{p}_{\mathrm{D}}$, both $\in \mathbb{R}^{L}$, indicate the transmitted power at each cell in reference signals (RS) (used for cell selection) and data channels, respectively.

Cell selection is based on average RS received power which can be calculated by means of the following expression:

$$
\mathbf{R}_{\mathrm{RS}}=\mathbf{G} \cdot \operatorname{diag}\left(\mathbf{p}_{\mathrm{RS}} \odot \mathbf{x}\right) .
$$

\footnotetext{
${ }^{1} \mathrm{~A}$ US patent application has been made for the multiobjective based CSO algorithm presented in this paper. Application no: 61/847,403; application date: 17 July 2013.
} 
The operator $\odot$ indicates Hadamard (pointwise) operations. Note that the vector $\mathbf{x} \in\{0,1\}^{L}$ indicates which cells are active and which ones are switched off. Hereafter, any $\mathbf{x}$ is referred to as 'a network configuration' or 'a solution' of the multiobjective optimization problem that is introduced shortly. Thus, the pixel $a$ ( $a^{\text {th }}$ row in $\mathbf{R}_{\mathrm{RS}}$ ) is served by cell $l^{\star}$ if:

$$
l^{\star}=\underset{l \in\{1,2, \ldots, L\}}{\operatorname{argmax}} \mathbf{R}_{\mathrm{RS}}(a, l)
$$

Note that the dependence of $l^{\star}$ on $\mathrm{x}$ has not been explicitly indicated. Based on (1) and (2), the binary coverage matrices $\mathbf{S}$ and $\mathbf{S}^{\mathbf{c}} \in \mathbb{R}^{A \times L}$ can be obtained. If $a$ is served by $l^{\star}$, then $\mathbf{S}\left(a, l^{\star}\right)=1 . \mathbf{S}^{\mathrm{c}}$ is the binary complement of $\mathbf{S}$. Note that the coverage pattern, implicitly defined in $\mathbf{S}$, is a function of $\mathbf{x}$.

The vector $\boldsymbol{\Gamma} \in \mathbb{R}^{A}$ corresponds to the spatial traffic distribution. Thus, $\boldsymbol{\Gamma}$ indicates the probability (in the event of a new a user) of each pixel having the user on it, and hence, $\boldsymbol{\Gamma}^{\mathrm{T}} \cdot \mathbf{1}=1$. Note that, if users are uniformly distributed in the coverage area, each element $\gamma_{a} \in \boldsymbol{\Gamma}$ is equal to $1 / A$. Since the traffic behavior is statistically well-known to the mobile operators, it is reasonable to assume that $\boldsymbol{\Gamma}$ is available. The assumption is commonly found in the CSO literature [5], [13], [15], [16].

In addition, it has been also considered, without loss of generality, that $\mathbf{p}_{\mathrm{D}}=\mathbf{p}_{\mathrm{RS}}$. In this manner, the vector $\boldsymbol{\Psi} \in \mathbb{R}^{A}$ representing the average SINR at each pixel is given by

$\mathbf{\Psi}=\left[(\mathbf{S} \odot \mathbf{G}) \cdot\left(\mathbf{p}_{\mathrm{D}} \odot \mathbf{x}\right)\right] \oslash\left[\left[\left(\mathbf{S}^{\mathbf{c}} \odot \mathbf{G}\right) \cdot\left(\mathbf{p}_{\mathrm{D}} \odot \mathbf{x}\right)\right] \oplus \sigma^{2}\right]$.

The operators $\oslash$ and $\oplus$ also indicate pointwise operations, $\sigma^{2}$ is the noise power. It is worth saying that the definition of $\boldsymbol{\Psi}$ describes ICI realistically as only the active cells are taken into account. Without loss of generality, it has been assumed that collisions (among active cells) occur with probability 1 as CSO schemes tend to concentrate traffic in few cells. Nevertheless, load factors $<100 \%$ can be introduced easily in the system model [18].

The $a^{\text {th }}$ pixel is in outage if the following conditions are not met:

- Minimum received power: $\mathbf{R}_{\mathrm{RS}}\left(a, l^{\star}\right) \geq P_{\min }^{\mathrm{Rx}}$.

- Minimum SINR: $\psi_{a} \geq \psi_{\min }, \psi_{a}=\boldsymbol{\Psi}(a)$.

The vector $\mathbf{H} \in \mathbb{R}^{A}$ contains the spectral efficiency of each pixel, where $\mathbf{H}(a)=\eta_{a}$ corresponds to the $a^{\text {th }}$ pixel. Spectral efficiency is computed as a non-decreasing function of the SINR. To capture the level of sensitivity with respect to the SINR variations, the Shannon's formula is considered. Thus, in order to take into account the coverage criteria and penalize solutions with coverage holes, the spectral efficiency of the $a^{\text {th }}$ pixel is computed according to the following rule:

$$
\eta_{a}=\mathrm{u}\left(\psi_{a}-\psi_{\min }\right) \cdot \mathrm{u}\left(\mathbf{R}_{\mathrm{RS}}\left(a, l^{\star}\right)-P_{\min }^{\mathrm{Rx}}\right) \cdot \log _{2}\left(1+\psi_{a}\right) .
$$

The function $\mathrm{u}()$ is the unit step function $(\mathrm{u}(z)=1$ if $z \geq 0$, and 0 otherwise).

The main target in any CSO scheme is minimizing energy consumption subject to QoS constraints. In this manner, two objectives are considered:

1) The minimization of the number of active cells $\left(f_{1}\right)$. The major part of the overall energy consumption in the network is proportional to the number of active cells [9], [14]. Thus, hereafter, $f_{1}$ is also called the Network Energy Level (NEL) and it is defined as follows:

$$
f_{1}=\mathbf{x} \cdot \mathbf{1}
$$

2) The maximization of the weighted network capacity $\left(f_{2}\right)$ : This metric is based on the expected value of the spectral efficiency at pixel level but including the effect of the traffic distribution $\Gamma$. Thus, for any given solution $x$, the metric is defined as follows:

$$
f_{2}=(B \cdot A)\left[\left[(\mathbf{H} \odot \boldsymbol{\Gamma})^{\mathrm{T}} \cdot \mathbf{S}\right] \odot \mathbf{n}\right] \cdot \mathbf{1} .
$$

Equation 6 deserves some comments. The vector $\mathbf{H} \odot \boldsymbol{\Gamma}$ contains the weighted spectral efficiency of each pixel as the nominal one is scaled by $\boldsymbol{\Gamma}$. The intuition is to give more importance to the $\mathrm{x}$ 's that are expected to provide better spectral efficiency to the zones with more concentrated traffic load. The constant $A$ corresponds to the number of pixels and it is used to normalize the obtained capacity to the reference case where all the pixels have the same probability, i.e., $\boldsymbol{\Gamma}(a)=1 / A \quad \forall a$. The vector $\mathbf{n} \in \mathbb{R}^{L}$ contains the inverse of the sum of each column in $\mathbf{S}$, i.e., the number of pixels served by each cell. It is assumed that each user is served by one cell at a time. This vector is used to distribute the capacity of each cell evenly over its coverage area, i.e., the bandwidth is shared equally by the pixels belonging to each cell. This improves the fairness in the long run similar to the proportional fairness policy that tends to share the resources equally among users as time passes. This fairness notion results in decreasing the individual rates as the number of users increases. This effect is also captured by $\mathbf{n}$ as the bandwidth per pixel is inversely proportional to the pixels associated with each cell.

\section{Multiobjective Problem Formulation}

In order to 1) capture the tradeoff between the number of active cells $\left(f_{1}\right)$ and aggregate network capacity $\left(f_{2}\right)$, and 2 ) obtain more than one single network configuration for each traffic profile $\boldsymbol{\Gamma}$, the use of multiobjective optimization has been investigated. The multiobjective optimization problem can be formulated as follows:

$$
\begin{aligned}
\begin{array}{r}
\operatorname{minimize} \\
\text { subject to: }
\end{array} & {\left[f_{1}(\mathbf{x}),-f_{2}(\mathbf{x})\right], } \\
& \frac{\left(\mathbf{v}^{\mathrm{T}} \cdot \mathbf{1}\right)}{A} \leq \kappa_{\mathrm{COV}}, \\
& \mathbf{x} \in\{0,1\}^{L}, \mathbf{x} \neq \mathbf{0} .
\end{aligned}
$$

Problem 7 defines the multiobjective framework considered herein. The objective functions in $7 \mathrm{a}, f_{1}$ and $f_{2}$, are defined by (5) and (6), respectively. Constraints $7 \mathrm{~b}$ and $7 \mathrm{c}$ correspond to the coverage criterion and the feasible set, respectively. The binary vector $\mathbf{v} \in\{0,1\}^{A}$ indicates the outage pattern associated to each solution $\mathbf{x}$. Therefore, if the $a^{\text {th }}$ pixel is in outage, $\mathbf{v}(a)=1$, and 0 otherwise. The parameter $\kappa_{\mathrm{COV}}$ is an operator-defined figure indicating how much (coverage) outage is allowed. 


\section{A. A bird's-eye view of multiobjective evolutionary optimization}

Multiobjective optimization is the discipline that focuses on the resolution of the problems involving the simultaneous optimization of several objectives such as $f_{1}$ and $f_{2}$. The target is to find a subset of good solutions $\mathcal{X}^{\star}$ from a set $\mathcal{X}$ according to a set of criteria $\mathcal{F}$, with cardinality greater than one. In general terms, the objectives are in conflict, and so, improving one of them implies worsening another. Consequently, it makes no sense to talk about a single global optimum, and hence, the notion of an optimum set $\mathcal{X}^{\star}$ becomes very important. A central concept in multiobjective optimization is the Pareto efficiency. A solution $\mathbf{x}^{\star} \in \mathcal{X}$ has Pareto efficiency if and only if there does not exist a solution $\mathbf{x} \in \mathcal{X}$, such that $\mathbf{x}$ dominates $^{2}$ $\mathbf{x}^{\star}$. The set $\mathcal{X}^{\star}$ of Pareto efficient solutions is called optimal nondominated set and its image is known as the Optimal Pareto Front (OPF). When a multiobjective problem is solved, it is unusual to obtain the OPF due to problem complexity; instead, a near-optimal or estimated Pareto front (PF) is found.

Problem 7 is a combinatorial problem that belongs to the class NP-complete, hence an optimal solution cannot be found in polynomial time. For this reason, heuristic-based algorithms are popular approaches in CSO. The so-called metaheuristics have become an active research field and their usage is gaining popularity as alternative to efficiently address problems in which the search space is very large [19].

In order to solve Problem 7 efficiently without relaxing the formulation, a strategy with the following features is required:

$\checkmark$ It must be able to find good solutions by efficiently exploring the search space.

$\checkmark$ It should operate efficiently with multiple criteria and a large number of design variables.

$\checkmark$ It should not have strong requirements on objective functions such as linearity, convexity, or differentiability.

Multiobjective evolutionary algorithms (MOEAs) [20] fulfill the previous requirements, and thus, their usage in CSO has been investigated herein. MOEAs are population-based metaheuristics that simulate the process of natural evolution. To be precise, a well-known MOEA was used in this study, the Nondominated Sorting Genetic Algorithm II (NSGA-II) [21]. NSGA-II is widely accepted as a reference in the field of evolutionary multiobjective optimization as it incorporates important features such as elitism, convergence, and distribution. An in-depth discussion of multiobjective and evolutionary optimization can be found in [19] and [20].

\section{B. Conceptual design and solution architecture}

Figure 1 illustrates the conceptual design of the proposed framework. The framework relies on the knowledge of the behavior of the traffic both in time and space. By means of different traffic distributions $\left(\boldsymbol{\Gamma}_{x}\right)$, the spatial component of the traffic at different moments of the day can be captured. These

\footnotetext{
${ }^{2}$ A solution $\mathbf{x}_{1}$ is preferred to (dominates in the Pareto sense) another solution $\mathbf{x}_{2},\left(\mathbf{x}_{1} \succ \mathbf{x}_{2}\right)$, if $\mathbf{x}_{1}$ is better than $\mathbf{x}_{2}$ in at least one criterion and not worse than any of the remaining ones.
}

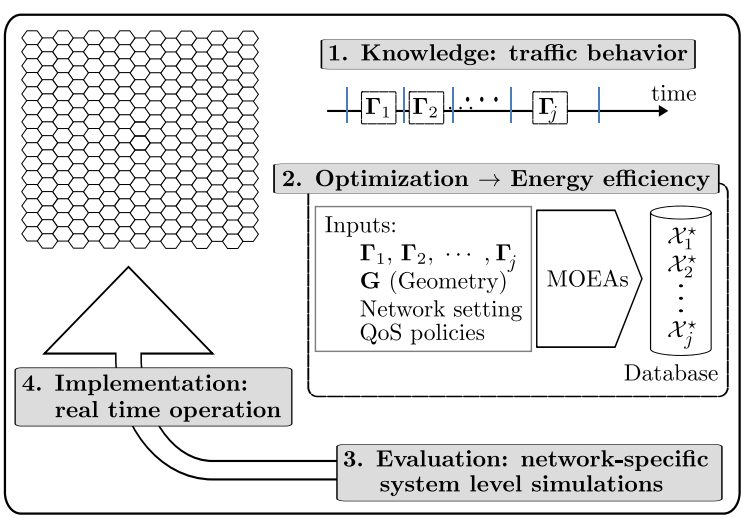

Fig. 1. Conceptual design of the multiobjective CSO framework.

patterns can be considered fairly constant during time intervals with arbitrarily small duration [5], [13].

Starting from the knowledge of $\boldsymbol{\Gamma}$, a set of Pareto Efficient solutions (with respect to $f_{1}$ and $f_{2}$, see (5) and (6)) can be obtained by means of a stochastic global search (MOEAs). It is important to say that $\mathcal{X}^{\star}$ is specific to $\Gamma$, and hence, a traffic pattern $\boldsymbol{\Gamma}_{j}$ results in its corresponding set $\mathcal{X}_{j}^{\star}$. The sets of nearoptimal solutions ( $\mathcal{X}_{j}^{\star}$ 's) can be evaluated offline by means of operator-specific system level simulations in which several QoS criteria, scheduling policies, and traffic load patterns can be considered independently. During the network operation, the network load and actual users distribution can be compared with the traffic profiles $\left(\boldsymbol{\Gamma}_{j}\right.$ 's) previously analyzed/stored by means pattern recognition techniques provided a basic level of coordination among cells. In this manner, a semidistributed/adaptive scheme that achieves significant energy consumption savings while guaranteeing the QoS can be obtained. The previous statement is supported by the results presented in the next section.

\section{Evaluation Setting and Results}

This section is composed of three parts. The first part explains the setting employed both for the multiobjective optimization procedure and system level simulations. The second part presents the results regarding the estimation of the set $\mathcal{X}^{\star}$, and finally, the last part shows a performance comparison between the proposed scheme and two previous proposals.

\section{A. Evaluation setting and simulation scenario}

The scenario corresponds to a dense deployment whose extension is comparable to a university campus. To be precise, the scenario is composed of 18 trisectorial sites with 54 cells and, without loss of generality, the sites are uniformly distributed following hexagonal geometry over a flat area of $1.55 \times 1.15 \mathrm{~km}^{2}$. The propagation model and the general setting is as indicated in [22] for urban microcellular deployments (UMi). Figures $2 a$ and $2 b$ show the average SINR map and a representation of the spatial traffic profile $\Gamma$.

Dynamic system level simulations are based on Monte Carlo experiments. The results compile statistics taken from 100 independent experiments each of which has a duration of $3600 \mathrm{~s}$. 


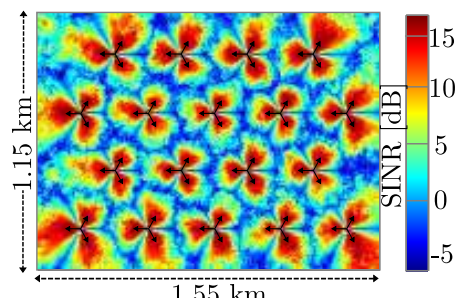

(a) Average SINR map

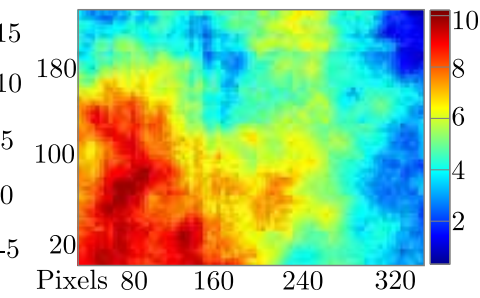

(b) Spatial traffic distribution: $\boldsymbol{\Gamma}$
Fig. 2. Test case for numerical evaluations.

TABLE II

Simulation Parameters and Evaluation Setting.

\begin{tabular}{|c|c|c|c|}
\hline \multicolumn{4}{|c|}{ General setting } \\
\hline Number of cells $(L)$ & 54 (trisec) & Inter-site distance & $300 \mathrm{~m}$ \\
\hline Pixels resolution & & Number of pixels & 71300 \\
\hline Transmit power $\left(P_{\max }\right)$ & $43.0 \mathrm{dBm}$ & System bandwidth $(B)$ & $5.4 \mathrm{MHz}$ \\
\hline Carrier frequency & $2.140 \mathrm{GHz}$ & Noise power $\left(\sigma^{2}\right)$ & $-174 \mathrm{dBm} / \mathrm{Hz}$ \\
\hline Shadowing std. dev. & $7.0 \mathrm{~dB}$ & Receivers noise figure & $7.0 \mathrm{~dB}$ \\
\hline Antennas height & $15 \mathrm{~m}$ & Antennas tilt & $19^{\circ}$ \\
\hline Radiation patterns & as in [22] & Antennas gain & $17 \mathrm{dBi}$ \\
\hline Minimum SINR $\left(\psi_{\min }\right)$ & $-7.0 \mathrm{~dB}$ & Sensitivity $\left(P_{\min }^{\mathrm{Rx}}\right)$ & $-123 \mathrm{dBm}$ \\
\hline \multirow{2}{*}{\multicolumn{4}{|c|}{$\begin{array}{l}\text { Coverage outage threshold }\left(\kappa_{\mathrm{COV} V}\right)<2 \% \\
\text { Subcarriers power }\left(\mathbf{p}_{\mathrm{RS}}, \mathbf{p}_{\mathrm{D}}\right) 5.67 \mathrm{dBm} / \mathrm{kHz}\end{array}$}} \\
\hline & & & \\
\hline \multicolumn{4}{|c|}{ Calibration of NSGA-II } \\
\hline Population size & 110 & Crossover prob. & 1.00 \\
\hline Type of variables & Discrete & Mutation prob. & $1 / L$ \\
\hline Termination criterion: & pervolume $\mathrm{g}$ : & $<0.001 \%$ in a block of & 10 gen \\
\hline \multicolumn{4}{|c|}{ Dynamic system level simulations } \\
\hline Target rate $\left(r_{\mathrm{T}}\right)$ & $250 \mathrm{kbps}$ & Target $\operatorname{QoS}(Q)$ & $98 \%$ \\
\hline $\begin{array}{r}\text { Us } \\
\text { Inter-arrival time: } \mathrm{Ra}\end{array}$ & distribution: & & 5007 \\
\hline $\begin{array}{l}\text { Inter-arrival time: Ra } \\
\text { Session time: Ran }\end{array}$ & om variable & ponentially distributed (1/ & $=0.075 \mathrm{~s})$ \\
\hline \multicolumn{4}{|c|}{$\begin{array}{l}\text { Random variable exponentially distributed }(1 / \mu=60.0 \mathrm{~s}) \\
\text { CSO }(Q \circ S \text { checking) interval: } 1 \mathrm{~s}\end{array}$} \\
\hline
\end{tabular}

At each cell, the scheduler assigns each user with a bandwidth such that the target rate $\left(r_{\mathrm{T}}\right)$ is satisfied. If the percentage of users that obtain a rate equal to $r_{\mathrm{T}}$ is greater or equal to the operator-specific target QoS $(Q)$ then the QoS policy is said to be fulfilled. Thus, in order to satisfy the maximum number of users, users are sorted based on their spectral efficiency and served accordingly. When there is not enough bandwidth to satisfy a user, the resource allocation finishes. The set of parameters used in simulations is provided in Table II.

The calibration of NSGA-II has been done according to the guidelines in [21]. Parameters used in simulations were obtained experimentally and are also shown in Table II.

\section{B. Estimation of nondominated solutions: The set $\mathcal{X}^{\star}$}

The resulting set of nondominated solutions is shown in Figure 3a. Note that the stochastic search performed by means of the evolutionary algorithm succeeds in estimating a full Pareto Front, i.e., one solution for each NEL $\left(\left|\mathcal{X}^{\star}\right|=L\right)$. As expected, the higher the number of active cells $\left(f_{1}\right)$, the higher the weighted network capacity $\left(f_{2}\right)$. As these solutions feature Pareto efficiency with respect to $f_{1}$ and $f_{2}$, the intuition in the design presented here is operating CSO strictly based on this small set of network configurations instead of searching

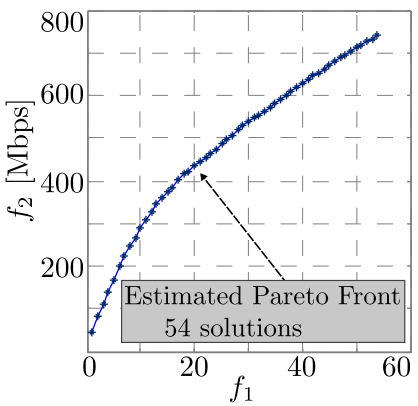

(a) Estimated Pareto Front

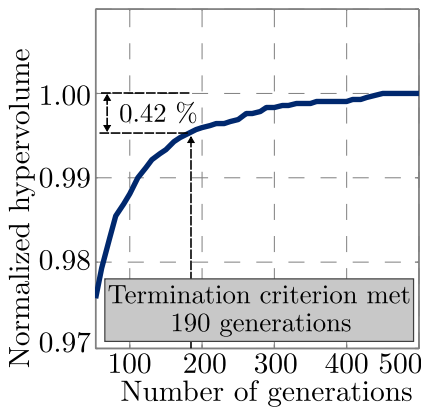

(b) Convergence pattern of NSGA-II
Fig. 3. Estimation of nondominated solutions: the set $\mathcal{X}^{\star}$.

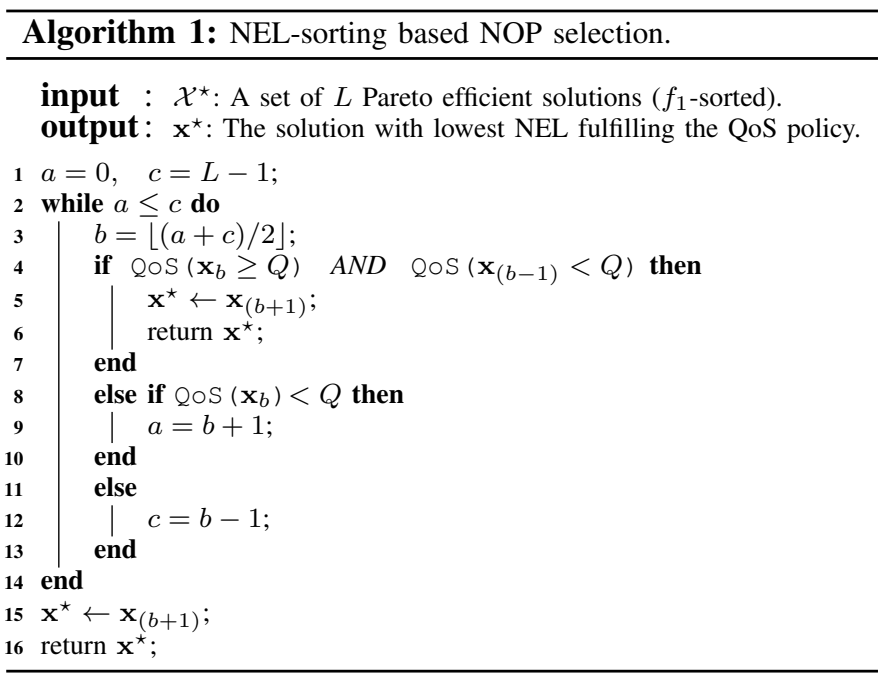

continuously in the whole domain whose cardinality is $2^{L}$, reducing the real-time complexity significantly. Figure $3 b$ illustrates the convergence of the evolutionary algorithm. It can be seen that the termination criterion (see Table II) is met, on average, after 190 generations.

However, in order to show the validity of this criterion, the search continued until 500 generations. It was found that additional 310 generations only provide a marginal gain of $0.42 \%$ in terms of the hypervolume indicator ${ }^{3}$.

Note in addition that, $\mathcal{X}^{\star}$ is found exploring only 100 . $\frac{190 \times 110}{2^{L}}=1.16 \times 10^{-10 \%}$ of the search space. This is due to the fact that $f_{2}$ guides the search to regions of $\mathcal{X}$, where the smaller set of solutions that have active cells providing better capacity to hot spots, are likely to be found.

In the next subsection, the solutions in $\mathcal{X}^{\star}$ are evaluated by means of dynamic system level simulations.

\section{System level simulations}

The set of solutions $\mathcal{X}^{\star}$ is evaluated through dynamic system level simulations considering two different approaches.

\footnotetext{
${ }^{3}$ The hypervolume indicator reflects the size of volume dominated by the estimated Pareto Front with respect to a reference point in the $n$-dimensional space. It is used to measure convergence and quality in multiobjective optimization. Additional information can be found in [23].
} 


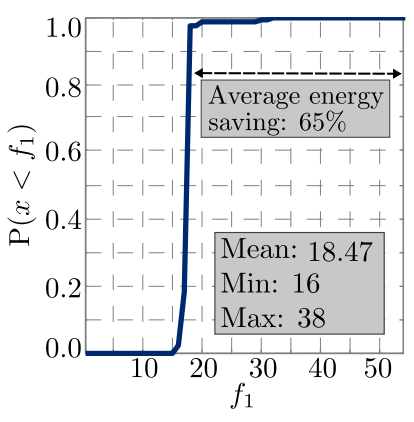

(a) Lowest NEL selection

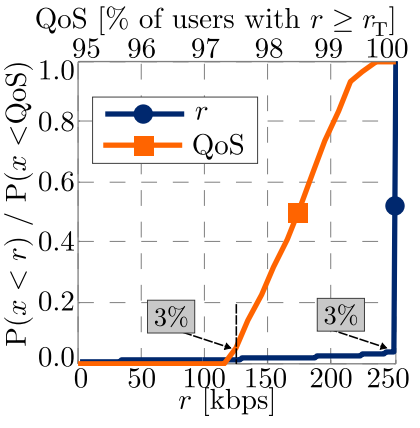

(b) QoS and users rate
Fig. 4. Selection based on NEL sorting: set $\mathcal{X}^{\star}$.

- Selection based on NEL sorting: At every QoS checking interval, all the solutions in $\mathcal{X}^{\star}$ are evaluated. The solution with the lowest value of $f_{1}$ that is able to satisfy the QoS policy is selected. To determine the winning solution at each time, a binary search based scheme can be used. Algorithm 1 shows the pseudo-code.

- Single NEL performance evaluation: One single solution is applied statically to study its performance without the possibility of switching to another solution.

Figure 4 shows the result corresponding to the selection based on NEL sorting. As it was mentioned, at every QoS checking interval, Algorithm 1 is executed to determine the solution with the lowest NEL that can provide the target rate to a fraction of users greater or equal than $Q$. Figure 4a shows the statistic of $f_{1}$ based on the solution that is selected at each time by Algorithm 1. It was found that $f_{1}$ is always in the interval $[16,38]$. However, the solution with $f_{1}=18\left(\mathbf{x}_{18}\right)$ is the one selected with high probability, and hence, the mean of $f_{1}$ becomes 18.47 . This implies that, on average, only 18.47 out of 54 cells need to be active to deliver the desired QoS when the traffic is distributed according to $\Gamma$ and the inter-arrival time is 75 ms. Thus, under that setting, Algorithm 1 achieves an average energy saving of around 65\% without degrading the QoS as it is shown in Figure 4b. The average user rates $(r)$ are around $50 \mathrm{kbps}$ to $250 \mathrm{kbps}$; only less than $3 \%$ the users have rates below the target rate $r_{\mathrm{T}}$.

The fact that our CSO scheme allows a marginal outage is a fundamental difference with respect to previous CSO proposals that are designed such that all users (without exception) need to be satisfied in order to move to a lower NEL, limiting drastically the possibility of saving energy. Thus, a marginal QoS threshold makes the design more robust and effective as the statistic of the user rates $(r)$ in Figure $4 \mathrm{~b}$ demonstrates.

In order to provide an additional perspective to the merit of the proposed framework, a performance comparison with reference schemes is conducted and analyzed. Reference schemes taken from the literature include: the Cell Zooming algorithm proposed in [14] and the Improved Cell Zooming scheme presented in [9].

Figure 5a illustrates the average NEL selection versus system load. Recall that the NEL is equivalent to the value of $f_{1}$

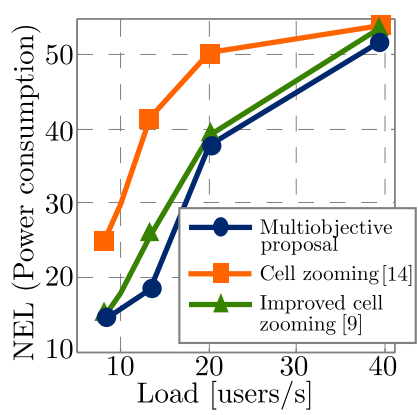

(a) NEL selection vs. system load

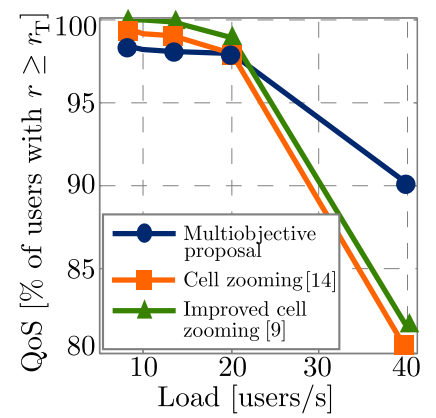

(b) QoS vs. system load
Fig. 5. Performance comparison among several CSO schemes.

for the solution selected at each QoS checking interval, and therefore, its mean value determines the energy saving that can be obtained, on average, with each scheme. As it can be seen, the proposed multiobjective framework outperforms the reference schemes, being the gains with respect to the closest benchmark (the improved cell zooming [9]) between $6 \%$ and $30 \%$. Besides, the good efficiency of the solutions in $\mathcal{X}^{\star}$ becomes evident when looking to the QoS provided to users. Figure $5 \mathrm{~b}$ shows that when the system goes to the overload region (40 new users per second in the network) the proposed framework improves the QoS by around $8 \%$ with respect to the improved cell zooming.

To complement this performance assessment, Table III provides additional performance figures including the average number of transitions and handover per QoS checking interval. In this manner, the performance-feasibility perspective is also considered. Transitions refer to the number of cells that should be switched on/off by selecting another network configuration. As it was mentioned, this is important because a high number of transitions results in an undesirable number of handovers due to the CSO process and a potential negative impact on the rest of the radio resource management functionalities. The first row shows the merit of the solution in $\mathcal{X}^{\star}$ with $f_{1}=18$, $\mathbf{x}_{18}$. Given that the NEL sorting selection indicated that such configuration is selected most of the time, it results interesting to see what happens if that configuration is applied during the whole simulation time. On the one hand, the degradation in terms of QoS with respect to the multiobjective proposal employing Algorithm 1 is minimal, and on the other hand, the proposed multiobjective framework achieves significant gains with respect to the benchmarks in terms of power consumption $(>50 \%)$, number of handovers $(>85 \%)$, and transitions $(>87 \%)$. These results clearly demonstrate the effectiveness and feasibility of the proposed scheme.

\section{Conclusions}

CSO is a framework that allows significant energy saving in cellular networks by switching off lightly loaded base stations. To do this, knowing traffic behavior both in time and space is a fundamental requirement.

The proposed multiobjective scheme is a new approach 
TABLE III

ADDITIONAL PERFORMANCE INDICATORS $(1 / \lambda=0.075 \mathrm{~s})$

\begin{tabular}{ccccc}
\hline Scheme & NEL & QoS & Handovers & Transitions \\
\hline $\mathbf{x}_{18} \in \mathcal{X}^{\star}$ & 18.00 & 97.81 & 0.00 & 0.00 \\
Alg. 1 & 18.47 & 98.74 & 7.14 & 0.31 \\
[14] & 41.45 & 99.05 & 57.56 & 3.47 \\
[9] & 25.36 & 99.87 & 80.14 & 7.47 \\
\hline
\end{tabular}

to analyze the CSO problem by 1) explicitly considering the spatial traffic distribution, and 2) introducing a weighted network capacity metric that prioritizes cells providing better capacity to zones of concentrated traffic.

System level trials confirm that the proposed scheme is effective and feasible as it achieves significant energy savings while minimizing the real-time complexity. This is possible due to the fact that the stochastic optimization procedure is performed offline and it results in a set of Pareto efficient solutions that exploit the tradeoff between energy requirement and aggregate capacity. A very particular feature of the proposed scheme is that real-time complexity is independent of the number of users as the optimization procedure is completely based on statistic information. Given that such traffic-profile-specific solutions can be stored in databases, lowcomplexity/semi-distributed implementations are feasible.

Although energy savings are, in general, network dependent, the proposed framework provides a flexible way to carefully fine tune parameters such as target QoS (rate) and coverage thresholds, such that operators can adjust the existing tradeoffs according to their needs and expectations.

\section{ACKNOWLEDGMENT}

The authors would like to thank Tamer Beitelmal and Davut Incebacak (Visiting Scholar) from Carleton University, and Dr. Gamini Senarath from Huawei Canada, for their valuable feedback.

This work is supported in part by Huawei Canada Co., Ltd., and in part by the Ontario Ministry of Economic Development and Innovation's ORF-RE (Ontario Research Fund - Research Excellence) program.

Mario García-Lozano is funded by the Spanish National Science Council through the project TEC2011-27723-C02-01 and by the European Regional Development Fund (ERDF)

\section{REFERENCES}

[1] Z. Hasan, H. Boostanimehr, and V. Bhargava, "Green Cellular Networks: A Survey, Some Research Issues and Challenges," IEEE Communication Surveys \& Tutorials, vol. 13, no. 4, pp. 524-540, Nov. 2011.

[2] T. Loozen, R. Murdoch, and S. Orr, Mobile Web Watch 2013, Accenture, 2013, Technical Report available online at: www.accenture.com.

[3] I. Humar, X. Ge, L. Xiang, M. Jo, M. Chen, and J. Zhang, "Rethinking Energy Efficiency Models of Cellular Networks with Embodied Energy,' IEEE Network, vol. 25, no. 2, pp. 40-49, Mar. 2011.
[4] M. Marsan, S. Buzzi, D. Ciullo, and M. Meo, "Optimal Energy Savings in Cellular Access Networks," in Proc. of IEEE International Conference on Communications (ICC 2009), Dresden (Germany), Jun. 14-18, 2009.

[5] E. Oh and B. Krishnamachari, "Energy Savings through Dynamic Base Station Switching in Cellular Wireless Access Networks," in Proc. of IEEE Global Telecommunications Conference (GLOBECOM 2010), Miami (United States), Dec. 6-10, 2010.

[6] M. Marsan, S. Buzzi, D. Ciullo, and M. Meo, "Switch-Off Transients in Cellular Access Networks with Sleep Modes," in Proc. of IEEE International Conference on Communications (ICC 2011), Kyoto (Japan), Jun. 5-9, 2011.

[7] L. Saker and S. Elayoubi, "Sleep Mode Implementation Issues in Green Base Stations," in Proc. of IEEE 21st International Symposium on Personal, Indoor and Mobile Radio Communications (PIMRC 2010), Istanbul (Turkey), Sep. 26-29, 2010.

[8] A. Bousia, E. Kartsakli, L. Alonso, and C. Verikoukis, "Energy Efficient Base Station Maximization Switch Off Scheme for LTE-Advanced," in Proc. of IEEE 17th IEEE International Workshop on Computer Aided Modeling and Design of Communication Links and Networks (CAMAD 2012), Barcelona (Spain), Sep. 17-19, 2012.

[9] F. Alaca, A. B. Sediq, and H. Yanikomeroglu, "A Genetic Algorithm based Cell Switch-Off Scheme for Energy Saving in Dense Cell Deployments," in in Proc. of IEEE Global Telecommunications Conference Workshops (GLOBECOM Wkshps 2012), Anaheim (United States), Dec. 3-7, 2012.

[10] T. Kang, X. Sun, and T. Zhang, "Base Station Switching Based Dynamic Energy Saving Algorithm for Cellular Networks," in Proc. of 3rd IEEE International Conference on Network Infrastructure and Digital Content (IC-NIDC 2012), Beijing (China), Sep. 21-23, 2012.

[11] S. Zhou, J. Gong, Z. Yang, Z. Niu, and P. Yang, "Green Mobile Access Network with Dynamic Base Station Energy Saving," in Proc. of ACM 15th Annual International Conference on Mobile Computing and Networking (MobiCom 2009), Beijing (China), Sep. 20-25, 2009.

[12] L. Saker, S.-E. Elayoubi, and T. Chahed, "Minimizing Energy Consumption via Sleep Mode in Green Base Station," in Proc. of IEEE Wireless Communications and Networking Conference (WCNC 2010), Sydney (Australia), Apr. 18-21, 2010.

[13] C. Peng, S.-B. Lee, S. Lu, H. Luo, and H. Li, "Traffic-Driven Power Saving in Operational 3G Cellular Networks," in Proc. of ACM 17th Annual International Conference on Mobile Computing and Networking (MobiCom 2011), Las Vegas (United States), Sep. 19-23, 2011.

[14] Z. Niu, Y. Wu, J. Gong, and Z. Yang, "Cell Zooming for Cost-Efficient Green Cellular Networks," IEEE Communications Magazine, vol. 48, no. 11, pp. 74-79, Nov. 2010.

[15] L. Sanchez and L. Munoz, "Energy Efficiency of Simple ON/OFF Scheme in Mobile Cellular Networks," Electronic Letters, vol. 46, no. 20, pp. 1404-1405, Sep. 2010.

[16] R. Li, Z. Zhao, X. Zhou, and H. Zhang, "Energy Savings Scheme in Radio Access Networks via Compressive Sensing-based Traffic Load Prediction," Transactions on Emerging Telecommunications Technologies, vol. PP, no. 99, pp. 1-11, Aug. 2012.

[17] S. Morosi, P. Piunti, and E. D. Re, "Sleep Mode Management in Cellular Networks: A Traffic Based Technique Enabling Energy Saving," Transactions on Emerging Telecommunications Technologies, vol. PP, no. 99, pp. 1-11, Jan. 2013.

[18] I. Siomina and D. Yuan, "Analysis of Cell Load Coupling for LTE Network Planning and Optimization," IEEE Transactions on Wireless Communications, vol. 11, no. 6, pp. 2287-2297, 2012.

[19] T. Weise, Global Optimization Algorithms - Theory and Application, 2nd ed. Self-published, 2009, online available at http://www.it-weise.de/.

[20] C. A. Coello, G. B. Lamont, and D. A. Van Veldhuizen, Evolutionary Algorithms for Solving Multi-Objective Problems, 2nd ed. Springer: Genetic and Evolutionary Computation Series, 2007.

[21] K. Deb, A. Pratap, S. Agarwal, and T. Meyarivan, "A Fast and Elitist Multiobjective Genetic Algorithm: NSGA-II," IEEE Transactions on Evolutionary Computation, vol. 6, no. 2, pp. 182-197, Apr. 2002.

[22] Radiocommunication Sector, Guidelines for Evaluation of Radio Interface Technologies for IMT-Advanced, ITU, 2008, Technical Report ITU-R M.2135.

[23] Q. Yang and S. Ding, "Novel Algorithm to Calculate Hypervolume Indicator of Pareto Approximation Set," Advanced Intelligent Computing Theories and Applications, vol. 2, no. 99, pp. 235-244, Jan. 2007. 